\title{
Prof. Fred Volkmar and Ben Popple: Yale Seminar on Autism: Autism and Dentistry
}

\author{
Yale Child Study Center, 2014, Itunes U
}

\section{Roy Rockman}

(C) Springer Science+Business Media New York 2014

\begin{abstract}
Autism affects 1 in 68 US children. It is more common than pediatric AIDS, diabetes, and cancer combined. As pointed out by Drs. Volkmar and Popple the major deficits that these children possess are difficulties in communication, social interaction, and sensory processing. These characteristics pose very unique challenges in maintaining dental health both at home and in the dental office. After an excellent discussion of the core deficits found in autistic children Dr. Popple continues to help define strategies aimed at preventive care and the etiology of dental disease. He emphasizes early intervention and the establishment of the dental home.
\end{abstract}

Despite all efforts by pediatric dentists to make the dental office comfortable for children, the sights, sounds, and smells of the dental office can create anxiety. This is particularly true of children with ASD who don't adapt well to changes in their environment or routine. In this presentation several suggestions are given in an attempt to anticipate these behavioral challenges and management techniques that will improve cooperation. The authors clearly point to the variability of the core symptoms in each individual diagnosed with ASD. Parental concerns sometimes revolve around the various dental materials utilized in preventive and restorative procedures. This issue is again addressed in the presentation. 